Carbon in Catalysis

\title{
Introduction
}

Carbon in catalysis has a long\{JÅntgen, 1986 \#3142\}\{Radovic, 1997 \#2938\} history covering diverse aspects of functions as support\{Rodriguez-Reinoso, 1998 \#2935\}\{Baker, 2004 \#306\}, poison\{Baker, 1995 \#2840;Baker, 1972 \#2995\} or active phase $\{\mathrm{Su}, 2010 \# 902\}$. The endless variability of structures arising from the electronic structure of the carbon atom gives rise to these many different functions\{Schlogl, 2008 \#198\}\{Schlögl, 2002 \#849\} in catalysis. It is well-known that carbon binds very strongly to itself giving rise to three hybrizations of its atomic electron structure: Whereas the linear sp hybrid (carbyne)\{Radovic, 2005 \#2871;Kasatoch.Vi, 1973 \#2915\} is of limited relevance here, the trigonal sp2 and the tetrahedral sp3 hybridizations are of key relevance to catalytic applications. In analogy to the wealth of structures in "organic chemistry" where any combination of these hybrizations is possible, the inorganic carbon chemistry is also quite involved due to the same mixing of trigonal and tetrahedral bonding\{McKenzie, $1996 \# 3130\}$. Traditionally one distinguishes graphite\{Simpson, 2004 \#204;Giannozzi, 2003 \#76;Alba, 2003 \#3302;Shimizu, 2002 \#3303;Letardi, 2002 \#3333;Atamny, 2000 \#3190;Alexandrou, 1999 \#1103;dos Santos, 1998 \#3354;Knop, 1996 \#572;Ugarte, 1995 \#2848;Steiner, 1995 \#3359;Ranish, 1993 \#3027;Ata, 1993 \#3067;Atamny, 1992 \#282;Schlîgl, 1992 \#3036;Nowakowski, 1992 \#3243;Kavanagh, 1988 \#2900;Batra, 1987 \#3187;McKee, 1984 \#3227;Schlögl, 1983 \#819;Moore, 1980 \#3054;Boehm, 1964 \#3012\} and diamond\{Casiraghi, 2006 \#3285\}\{Kraft, 2008 \#1048;Roddatis, 2002 \#739;Martin, 1996 \#147;Sappok, 1968 \#2910\} as the inorganic carbon allotropes with pure trigonal planar and tetrahedral connectivity respectively.

In the last decade many molecular or extended versions of carbon have been synthesized or are subject to scientific interest. These samples are collected under the term "SCA" or synthetic carbon allotropes. In these compounds carbon bonding of the traditional allotropes that fulfil the definition of a structural modification of a single element is intermixed with heterobonds between carbon and other elements. It would be desirable if the community could adhere to the IUPAC definitions of carbon terminology\{Rodriguezreinoso, 1989 \#2956\} rather than invent new terms being partly in conflict with these definitions. 
Defective forms of carbon called soot or black carbons were known earlier to be of complex mixed hybridisation. Carbon systems exist with large fractions of sp3 centres in sp2 matrices. Such carbons\{Robertson, 1992 \#3132;Robertson, 1991 \#3131\} are superhard, form layers and are chemically highly resistant. Few attempts were made so far to exploit these materials in catalysis beyond the use of "glassy carbon " $\{$ Zeng, 2006 \#3211;Schmidt, 1998 \#1112;Yang, 1994 \#3365\} in electrocatalysis. Rules for the designation of carbons were defined\{Rodriguezreinoso, 1989 \#2956\}, without finding, however, strict application in the fast -growing literature. With the advent of nanostructured carbons and with the insight into non-thermal synthesis methods\{Titirici, 2008 \#221\}\{Paraknowitsch, 2009 \#173;Thomas, 2008 \#220\} of carbon the number of inorganic carbon forms has exploded making a complete systematic description impossible, partly also because of the demanding task of a full characterization\{Tessonnier, 2009 \#922;Muller, 2006 \#2664;Braun, 2005 \#3255;Pasieczna, 2004 \#3375;Raymundo-Pinero, 2002 \#2708;Bockhorn, 2002 \#3136;Belz, 1998 \#2737;Contescu, 1997 \#3325;Schlögl, 1996 \#813;Stîckli, 1995 \#3144;Yang, 1994 \#3365;Henschke, 1992 \#480;Werner, 1992 \#3104;Audier, 1981 \#3167\} of the complex structures. An overview over some here relevant forms is given in Figure 1.

The most frequent encounter of catalysis science with carbon is not in any beneficial form but rather as phenomenon of deactivation in hydrocarbon transformations. The forms of carbonaceous deposits, of soot, of coke ("white" and "black") are polymers of hydrocarbons with low carbon-to hydrogen ratios below 1 belonging thus to the large family of precursor of inorganic carbons characterized by a carbon to hydrogen ratio of below 0.1 . The precursor state of these unwanted carbons is often reported when during oxidative regeneration some of the carbon converts from reactive in non-reactive forms. During oxidative removal of the precursor forms the strong release of combustion energy provides the driving force for non-oxidative dehydrogenation and condensation of remaining precursor making the resulting polymer less reactive for oxidation. Control of the exothermicity of the regeneration can minimize this unwanted carbon synthesis processes. It should also be noted that in cases of transition metal active phases being present on the catalyst some the carbon precursors can dehydrogenate and dissolve in the metal particles (notably in $\mathrm{Pd}, \mathrm{Ni}, \mathrm{Fe}$, Co catalysts) causing the formation of solid solutions or sub-stoichiometric carbides and thus transforming the catalytic properties 
of such systems. The mechanism of carbon condensation starts from C2 or C3 olefines forming ring structures\{Fridman, $2010 \# 2447\}$ to which further olefins can be added creating sp2 networks of carbon. Multiple ring closure defects create structural reactivity leading to interconnections of graphene units and thus to 3 dimensional solids with mixed hybridisations of sp2 and sp3 connectivity ("hard cokes"). Hydrogen terminating atoms render these polymers chemically inert and stable against oxidation at elevated temperatures. When the condensation conditions allow for a slower growth and sufficient hydrocarbon is available the products undergo re-arrangements of the initial ring closure defects and form pure sp2 containing graphene units covering large fractions of a catalyst surface. An example of such a condensation product is shown in Figure 1. Here a vanadium-alumina catalyst\{Vass, $2008 \# 230\}$ used for oxidative dehydrogenation was operated under such productive conditions that the resulting olefine butene underwent dehydrogenation and condensation in competition to desorption which would be the expected process at $873 \mathrm{~K}$ operation temperature. The image reveals that the highly active catalyst is fully covered with a graphene layer giving rise to the conclusion that not the initial oxide catalyst is active but rather the curved graphene layer. The corresponding NEXAFS spectrum acquired in-situ\{Vass, 2008 \#230\} indicates a strongly graphitic nature of the deposit (see also next sections). The active carbon is distinctly different in its geometric and electronic structure from soot deposits with much less sp2 content as could also be verified with in-situ spectroscopy. The example reveals that in some cases (see examples below) the unwanted debris of carbon can restructure under operation conditions into an active catalyst\{Vass, 2008 \#230\} taking over the desired catalytic function from the initially present oxides or metallic active phase. 

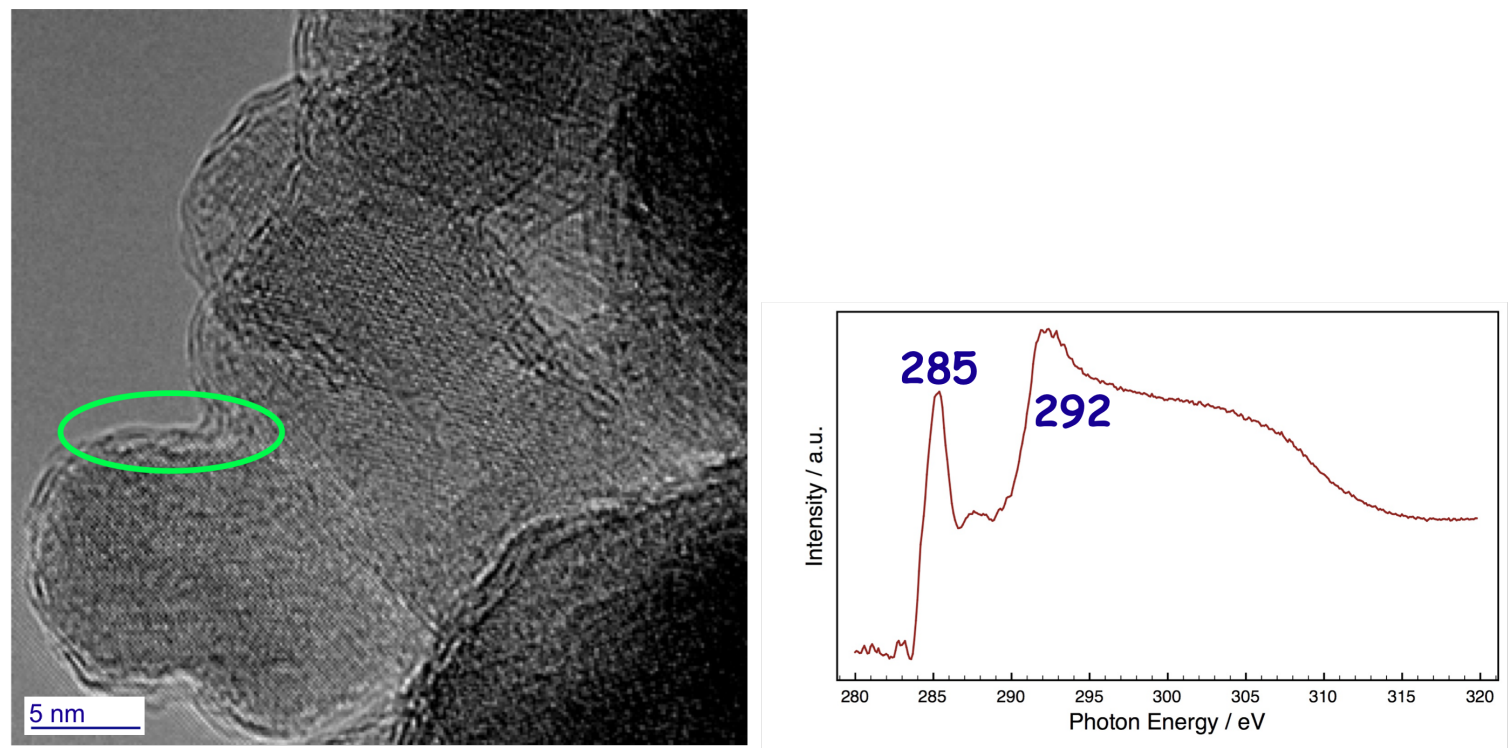

Figure 1: TEM image of a 3.5\% vanadia on alumina catalyst used for butene oxidative dehydogenation at 873 $K$. The oxide nanostructure is fully encapsulated with carbon revealing large fractions of coplanar graphene units together with some fullerenoidic carbon and some twisted carbon bands. The carbon K edge NEXFAS spectrum acquired in-situ under comparative conditions reveals the high sp2 content of the carbon as indicated by the features annotated with photon energies arising from the $\pi^{*}$ and $\sigma^{*}$ transitions respectively. From ref.\{Vass, 2008 \#230\}

The purpose of this article is to extract some concepts standing behind the application of carbon in catalysis rather than giving an account of the successful application examples. The reader interested in these aspects is referred to an excellent monograph\{Serp, 2009 \#3910\} covering this subject in exhaustive manner. The organization of this article is as follows. Some considerations on the electronic structure of carbon is used as foundation for reactivity considerations. Then follows a description of catalytically relevant forms of carbon and some comments on the complex and controversial issue of surface functional groups and their analysis. Several case studies reveal the level of functional analysis reached and introduces some models of reactive sites. A generalization of the concepts of application of carbon in catalysis rounds off this account of a rapidly moving field.

\section{The electronic structure of nanocarbon}

The vast complexity of the carbon material family provokes a phenomenological approach towards exploiting its potential in catalysis. A large number of applications deal with the anchoring of metal or oxide particles on various forms of carbon. A considerably smaller number of reports describe the use of carbon as a catalyst in its 
own right whereby mostly the active sites are used that arise from the rich functionalization chemistry of carbon.

A more rational approach towards carbon in catalysts begins with the study of electronic structures of carbon that allows predicting chemical reactivity and target structures useful in catalysis. Unfortunately, the analysis of the electronic structure is not straightforward as the standard chemical spectroscopy tools like X-ray structure determination, IR and NMR are of limited use only and UHV-based electron spectroscopy is difficult-to-apply on powder samples that cannot be purified in a reproducible manner.

A useful tool\{Castiglioni, 2006 \#2822;Dillon, 2004 \#3039;Jager, 1999 \#2732\} in discriminating the type and intermixing of hybridization is RAMAN spectroscopy. The Raman spectrum of a pure sp2 carbon system consists of a strong line arising from the C-C stretching vibration in the molecular picture or from the G-band in the phonon band formalism\{Pimenta, 2007 \#3911\}. This band is sharp at $1587 \mathrm{~cm}-1$ for planar systems and gets split and shifted for curved systems with a shift range up to about $1630 \mathrm{~cm}-1$. Defects in the perfect hexagonal symmetry cause the anti-symmetric vibration being RAMAN forbidden to become active giving rise to two resonances at around $1350 \mathrm{~cm}-1$ and $1600 \mathrm{~cm}-1$ designated as D-bands in the literature. Superimposed on this set of bands are additional signals from localized states where the RAMAN signal appears from molecular excitations of aromatic ring stretching vibrations and from carbonheteroatomic bonds. These bands broadening the whole spectral range between the D and G bands exhibit resonant features (intensity depending on excitation energy) whereas the genuine extended $\mathrm{sp} 2$ features are non-resonant features. Theoretical treatment reveals that the distinction between clear categories of molecular and extended structures is too simple and that different ordering states of carbon exhibit gradual mixtures\{Castiglioni, 2006 \#2822\} of vibrational excitation. Fitting routines were developed\{Knauer, 2009 \#567\} to derive in a pragmatic phenomenological approach the degree of hybridization and the contribution from molecular species to mixed nanocarbon systems. Edge defects relevant for the chemistry of carbon can be identified by RAMAN spectroscopy\{Pimenta, 2007 \#3911\} as well as structural ordering in an integral way and complementary to diffraction methods\{Sanchez-Cortezon, 2000 \#756\}. A characteristic experiment is shown in Figure 2. By ball milling natural highcrystalline graphite was defected by breaking the flakes both in thickness and size. The 
number of defects indicated by the $\mathrm{D}$ band features rose dramatically on the expense of the feature characteristic of the G mode.

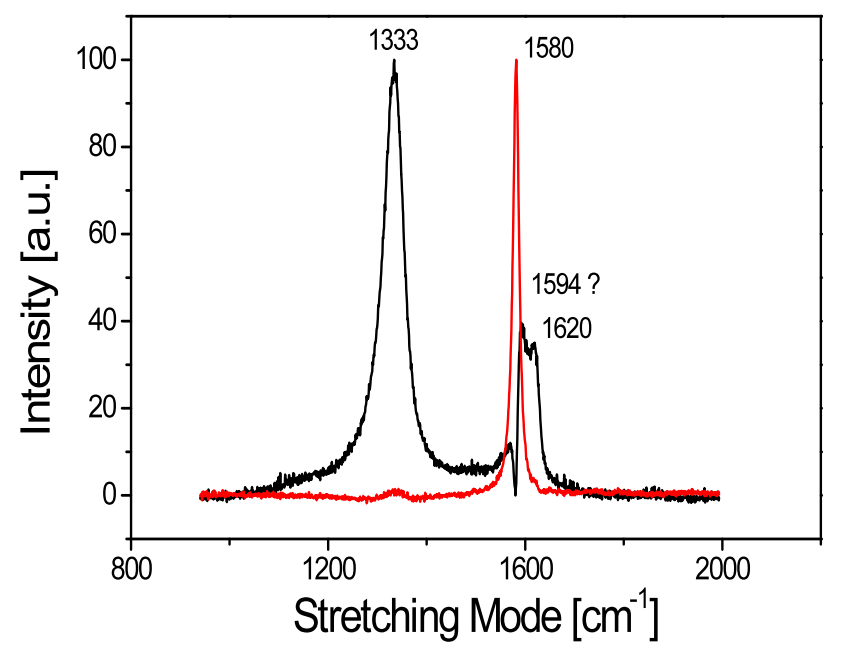

Figure 2: RAMAN spectra of fresh (red) and ball milled (168 h in air) natural graphite. The indicated shift (?) of the $G$ band may be accounted for by massive bending of the tubostratically disordered garphee units following milling and incorporation of planar defects (sen by TEM and reactivity see below). The dual lines of $D$ and $D^{`}$ can be seen well.

The valence electronic structure of here relevant carbons may be discussed in terms of the parent graphite structure. Graphite is a two-dimensional solid. The corresponding material is graphene,\{Rao, $2009 \# 2055\}$ consisting of a single layer of graphite. It is noted that graphene structures were discussed in carbon chemistry long before arouse \{Wertz, 1994 \#2860\}\{Boehm, 1997 \#2902;Boehm, 1958 \#2912\} the actual interest. Graphenes interact through weak dispersive forces giving rise to the crystal structures of graphite\{Boehm, $1964 \# 3012\}$ (hexagonal and rhomboherdal). The electronic coupling between graphene layers is so weak\{Dresselhaus, 1992 \#3080\} that graphite is a semi-metal, exhibiting electronic metallic conductivity only within the graphene sheets and semiconducting poor conductivity perpendicular to it. The electronic anisotropy as measured by resistivity can reach 4 orders of magnitude.

This electronic anisotropy causes also chemical anisotropy: on the basal planes we expect metallic properties with Lewis base chemistry whereas at the edge sites and prism face of graphite we expect insulating behaviour and Lewis acidic behaviour. The latter sites are coordinatively unsaturated and form in any atmosphere immediately surface functional groups by activating and binding hydrogen, oxygen, nitrogen or 
halogens. In this way the electronic anisotropy translates\{Schlögl, $2008 \# 805\}\{$ Schlögl, $2002 \# 849\}$ into an acid-base chemistry at prism sites and simultaneously the chemistry of a noble metal on the predominating basal planes.

The strong inter atomic bonding of carbon sp2 sites strongly reduces the reactivity of the basal planes as delocalized metallic electrons are existing there but with unusual high binding energy\{Carlsson, 2006 \#38\}. Thus, redox reactions are difficult but not impossible as a small number of electrons exhibits high enough energy\{Giannozzi, 2003 \#76;Shimizu, 2002 \#3303;Sorescu, 2001 \#3269\} to activate adsorbed molecules. The electronic structure is characterized by four bands\{Batra, 1987 \#3187;0oi, 2006 \#3009\} two of them with angle integrated energies\{Pantin, 2006 \#3007\} of about 13 and $7 \mathrm{eV}$ representing the $\sigma$ - symmetric orbitals of the sp2 system and two bands at about 3 and $1 \mathrm{eV}$ representing the $\pi$-symmetric states. Only the top valence band touches the border of the Brillouin zone at the $\mathrm{K}$ point and thus allows for a minute band overlap into the conduction bands. The angle-integrated density of states is very small exhibiting bare any feature at the Fermi level. This leads to the described poor reactivity of the basal plane.

For nanostructures the following consequences arise. Depending on the orientation of a surface as basal (nanotubes) or prismatic (herringbone fibres) the surface chemistry is either metallic or that of an oxide but without metal cores. This opens the possibility to perform metal-free catalysis\{Yang, 2004 \#3385\} with carbon provided the correct functionalization is applied. In Figure 2 some typical surface structures of nanocarbons are shown. It should be noted that these are prototypical simple morphologies with many more complex shapes being synthesized intentionally or unintentionally. In Figure 2 (C) a common problem with CVD carbon nanostructures is shown: the surface of the intended nanocarbon is covered by molecular polymeric species arising from pyrolytic condensation onto the catalytically formed nanocarbon. This frequently overlooked phenomenon renders the chemical properties of quite different nanocarbon structures apparently very similar: the anisotropy is lifted and replaced by the reactivity of the defect structure being more prone to substitutional chemistry and to gasification than the underlying parent nanocarbon. 

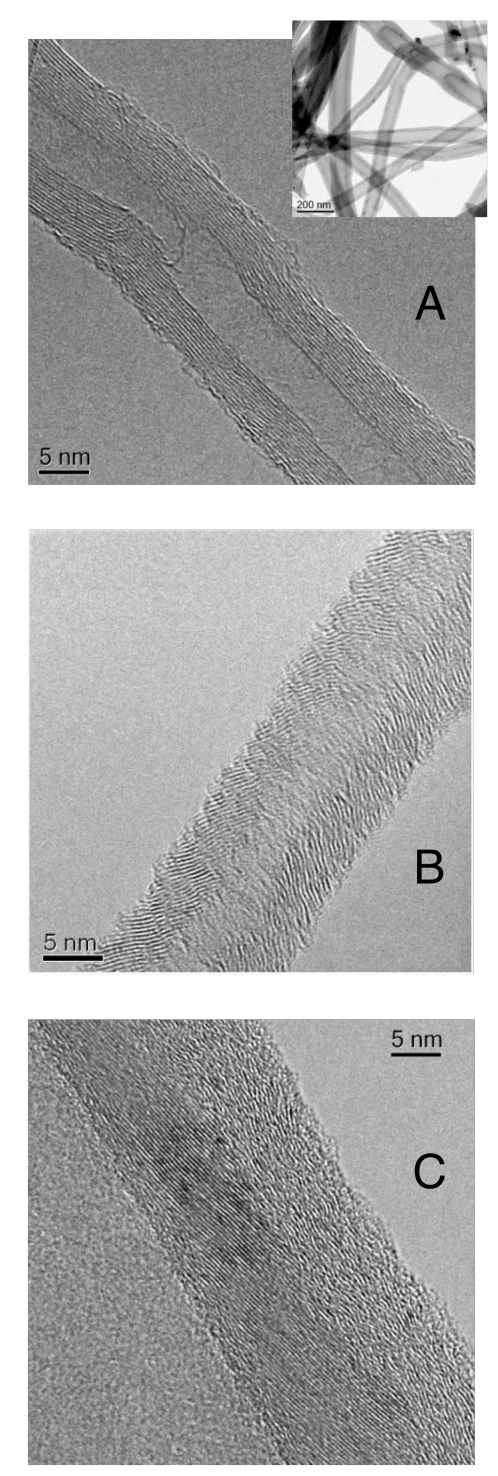

Bending of the graphene sheets to rounded\{Saito, 1992 \#3316\}\{Sanchez, 1999 \#2896\} or spherical objects (tubes, onions) leads to strong modification of the top of the valence band; the Brillouin zone changes and the dispersion of the electronic states my not touch the zone edge any more. In chemical terms this leads to localisation of the electrons in poly-olefinic double bonds giving rise to typical reactivity of such bonds coordinating metals and activated organic molecules (Diels Alder reactions). The size of a nanocarbon will change the surface-to-bulk atom ratio as well as the basal-to prism face ratio and thus modify the chemical reactivity more drastically than known for isotropic nanoparticles.

The low density of states leads to a much larger sensitivity of the electronic structure to such phenomena than seen in metallic systems, where the density of states approaches 
the number of atoms in the particle exhibiting hence a high density of mobile electrons and consequently a reduced sensitivity to geometry.

The strategy to strongly modify the chemical reactivity of nanocarbon lies in modifying the ratio between basal and prism faces. Besides macroscopic defecting large crystals by tribochemistry\{Yang, $2000 \# 2729$ \} or by selecting small graphene units into nested structures\{Joseph, 1983 \#2978;Audier, 1981 \#3165\}, the creation of point defects in basal plane is a highly effective way\{Peres, 2006 \#3008;Atamny, 1999 \#2730\} of doing so. Removing only one atom from the plane creates the so-called Stone-Wales defect leading to several sites of prismatic reactivity locally in a plane of inertness for chemical bonding. This is illustrated in Figure 3
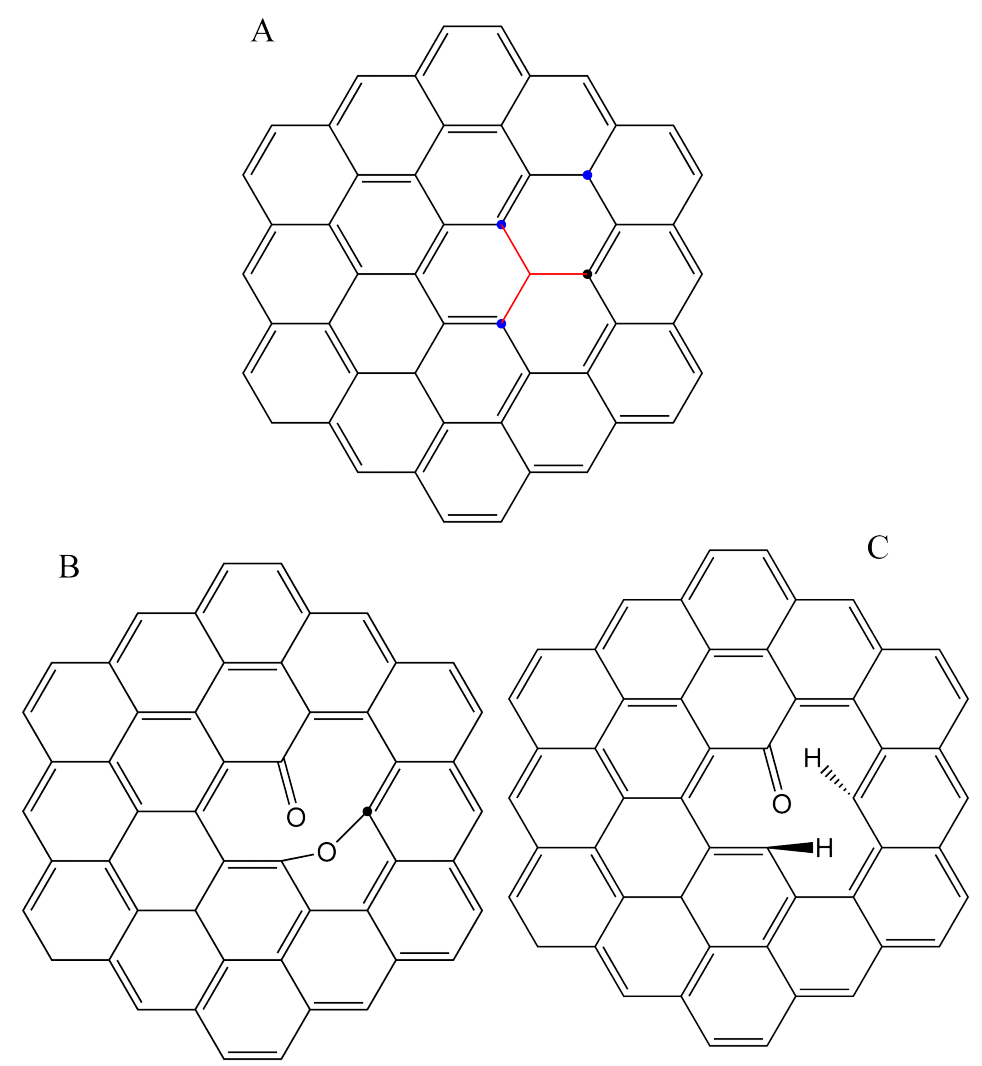

Figure 3: Representation of a single atom defect (A) and its resulting localized charges (blue). In (B) the stabilization is indicated through oxygen functional groups and in (C) an alternative with hydrogen and oxygen groups from a water molecule.

The resulting radical-containing defect saturates quickly with ether an oxygen molecule or a water molecule to the structures shown in Figure 3. The process will enhance the reactivity of graphene as both electron inductive effects and the bending of the initially 
flat graphene containing now a defect will re-hybridize the electronic system and create localized charges acting chemically as double bonds and forming local states near the "Fermi edge" in the band structure formalism.

This is shown in Figure 4 top as experiment and in Figure 4 bottom as result from theoretical\{Carlsson, 2006 \#38\} calculations. The top spectra show He I UPS data from a pristine HOPG with the expected very small density of states near the "Fermi edge" and the drastic change occurring from sputtering the surface with oxygen followed by annealing in vacuum. The rather smooth increase of the spectral curve with increasing binding energy reveals that the increase in spectral weight is due to localized states and not to free electrons. This is in good qualitative agreement with calculations of a model defect of the structure as shown in Figure 3 (B); additional states occur below the maximum of the highest $\pi$-symmetric band showing its maximum at about $3 \mathrm{eV}$ as discussed above. The multiplicity of defects arising from sputtering HOP cannot be removed by annealing and precludes thus a more detailed modelling of the electronic structure. For the purpose of describing the chemical reactivity of point defects the results may be considered sufficient to understand the concept of reactivity control outlined here.
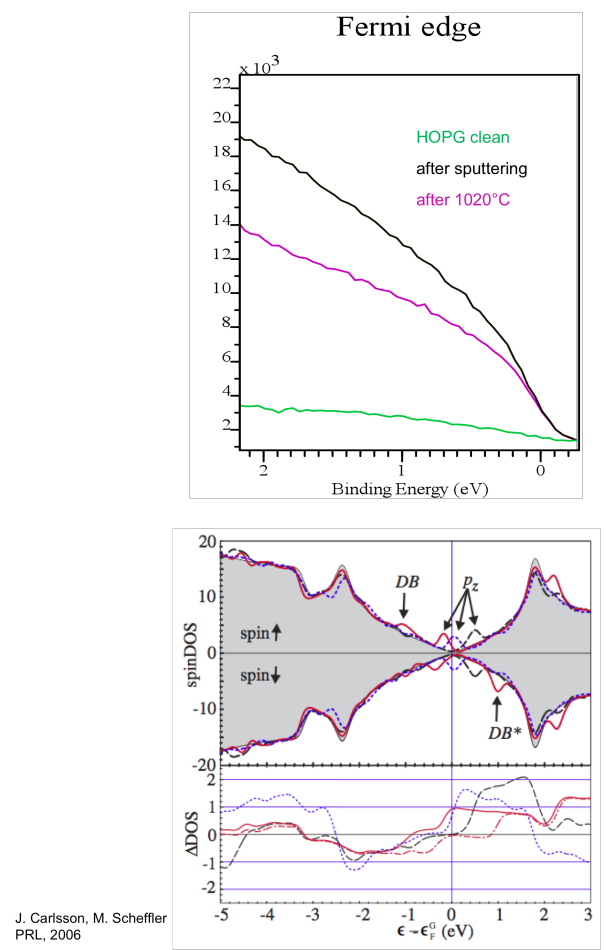
Figure 4. Effect of point defects on teh electronic structure of graphite (graphene). Top: UPS He I (21.22 eV) of a pristine HOPG surface (green) and aftre sputtering with $0.5 \mathrm{keV}$ for $1 \mathrm{~min}$ in an Ar/oxygen mixture (black) followed by UHV anealing at $1273 \mathrm{~K}$ (magenta). The bottom Figure shows results of theoretical modelling of a Stone-Wales defect saturated with the most stable configuration of oxygen shown in Figure 3 (B).

In Figure 5 we compare the electronic structures of planar and roled garphene as sensed with the anti-bonding states or conduction bands. The relevant spectroscopies EELS and XAS follow the same physiacl principle but use diffrent exciatation sources allowing to classify XAS as surafce-sensitive probe and EELS as bulk-sensitive probe. The direct comparison of the results $\{\mathrm{Su}, 2003$ \#901;Mitterbauer, 2003 \#1136\} as done in Figure 5 allows thus to estaimate the distribution of features with respect to the volume of the sample under study. Using an electron microscope for EELS allows further to visually inspecting the structural integrity of the saplme under study.

Following earlier asignments\{Braun, 2005 \#3255\}\{Itchkawitz, 1995 \#517;Atamny, 1992 \#18\}\{Francz, 1996 \#2898;Schedelniedrig, 1995 \#2820\} of the respective spectral features we see that the $\pi *$ features are narrow lines with a slight shift to higher photon energies for the CNT sample. The features above the pre-edge arise from defects and hetreo-elements. Diffrence spectra not shown revela clearly that the spectral properties of the $\pi^{*}$ are differnet for HOPG (narrow, symmetric) and CNT (wider, shifted) in agreement with the effect of re-hybridization of the sp2 hybrids by bending the graphene. This efect is, however, much smaller than the efefct $f$ disturbing the carbon electronic structure by forming carbon hydrogen and carbon-oxygn bonds at defects as sen by the unresolved features above $285 \mathrm{eV}$. These effects are comparabe to string rehybridization when sp3 bonds intermix with SP2 bonds. This case occurs for the hOPG sample where the oxygen EELS reveals the absence of carbon-hetreobonds (Fufure 5, bottom). Surface C-H contributes only little\{Schedelniedrig, 1995 \#2820\} to the signal. Notably the diffrence in electronic structures of curved and planar graphene is much smaller than the effects introduced into the graphene at edges and at point defects. The spectral range between the $\pi^{*}$ and the $\sigma^{*}$ derved-sates is here most sensitive. The wellresolved data of Figure 5 show more clearly than earlier lietrature data how the lowlying electronic states around the „Fermi egdge“ are perturbed by chemicaly relevant termination and defect states in full agreement with the description above. 

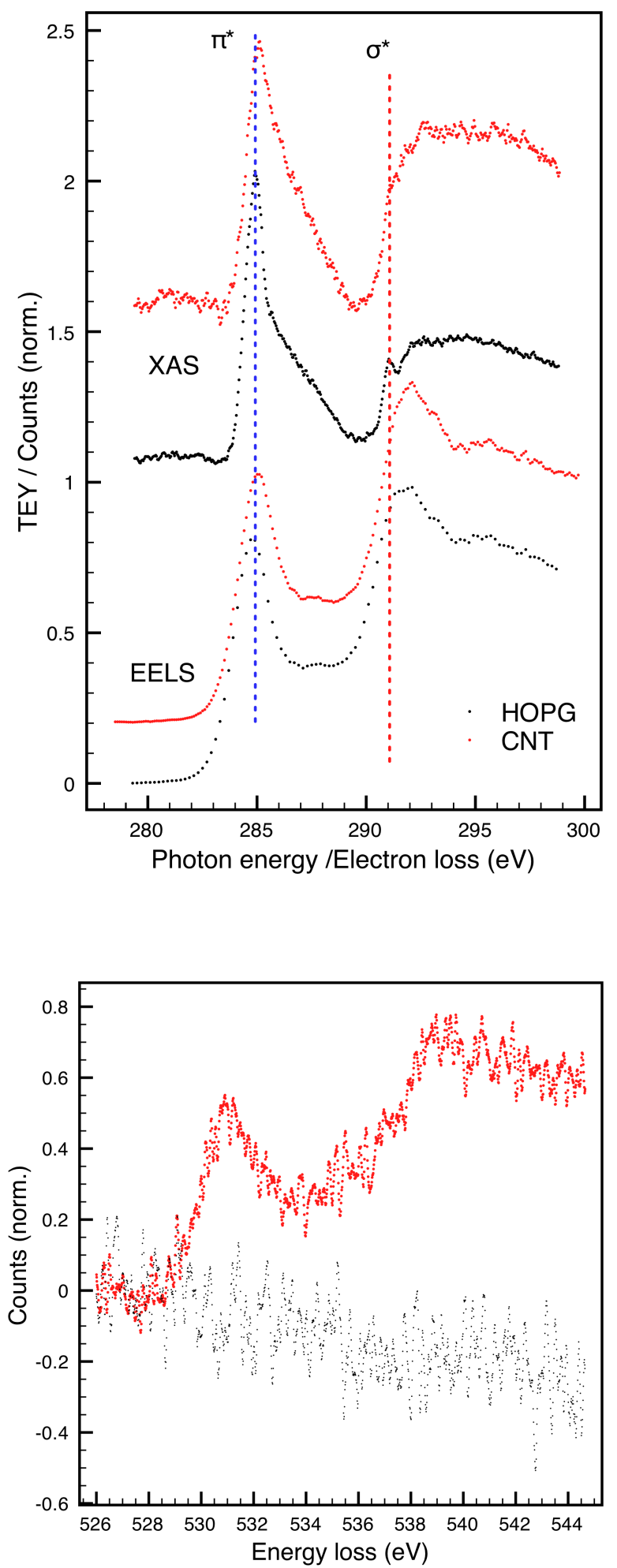

Figure 5: Compariosn of the electronc structure of HOPG graphite (black) and graphitized MWNT (red) in XAS and EELS. The top panel compares the carbon-derived features, whereas the bottom plot reports the oxygen-k edge features as measured by EELS. The vertical lines denote the lowest energy features from states of carbon $\pi$ and $\sigma$ symmetric states. The EELS specta were measured without aniotropy contributions at 
magic angle conditions. The XAS spectra were measured at 20 deg angle of incidence maximising the sensitivity for the $\pi *$ features.

It is evdent from Figure 5 that manipulating the surface functionalization on graphene both in planar and curved form exhibits a means of strongly aletring the local electronic structure. Active sites for cataylssi are thus amenable to controleld modification. It also occurs from this discussion that such sites are not frequent and thus an inherent weakness of carbon-derived cataylsts will be a low density of active sites. Attempts to greatly enhance this number will have t paid for with a strong reduction of the inetrgrity and stability of the materail that is guarn

The concept of heterogeneous metal-free catalysts

Support effects

Analysis of NC

Synthesis: how available are NC?

Synthesis of NC

The synthesis of nano-structured carbons is a critical issue for a mass application as represented by catalysis. It is evident that methods for the synthesis of abundant and suitable $\{\mathrm{Su}, 2008 \# 2635\}\{$ Tessonnier, 2009 \#922\}NC are pre-requisites for an application in chemistry. In addition, it must be stressed that loose nano-carbons are of little use\{Su, 2005 \#2681\}\{Vieira, 2002 \#232;Delgado, 2006 \#393\} in applications where transport limitations of reactants play a critical role. Also possible health and safety issues $\{\mathrm{Su}, 2008 \# 898\}\{$ Abdullah, 2009 \#2615\} of nano-structured materials in general and of $\mathrm{NC}$ in particular require a safe way of structuring carbons in mesoscopic dimensions by other means than mechanical compaction. 
Variety of NC in catalysis

The seamless combination of sp2 and sp3 coordinated carbon caters for an infinite amount of structural variants $\{$ Schlögl, $2008 \# 805\}$ of NC. many of them are realised in practical synthesis of carbon, not all of them are properly characterised and only few of them are useful in heterogeneous catalysis.

We first exclude the many variants of NC generated unintentionally during carbon deposition on catalysts. These "soot" type deposits are of complex\{Ebert, 1988 \#2962;Lahaye, 1990 \#2952;Belz, 1997 \#2818;Homann, 1998 \#2932\} structure. A significant content on heteroatoms and a quite limited size of their basic structural units (BSU) render them quite different\{Lange, 1988 \#2957;Karge, 1996 \#2939\} form black smoke soot\{Calcote, 1981 \#2985;Bockhorn, 1987 \#3138;Lahaye, 1990 \#2952;Werner, 1993 \#981;Schlögl, 1996 \#813\} synthesised as pigment or generated during incomplete fuel combustion.

The “catalytic soot"\{Clauss, 1957 \#2913;Mattevi, 2008 \#2798\}\{Macia-Agullo, 2005 \#609\}\{Atamny, 1998 \#2816;Boehm, 1994 \#2903;JÅntgen, 1986 \#3142;Karousis, 2010 \#2450;Lisovskii, 1994 \#2812;Sherigara, 2003 \#3042;Su, 2010 \#1070;Vrieland, 1991 \#2813\} can play multiple roles such as site blocker supporting selective reactions, as active sites or as poisons preventing access of reactants to active sites. In dehydrogenation reactions it was shown\{Vass, 2008 \#938;McGregor, 2010 \#1067\} that carbon deposits can exert several desired\{Sakurai, 2000 \#2806\} and undesired\{Kuhrs, 2001 \#2711;Schule, 2007 \#2648\} functions according to their structures being not under control of the catalyst user. It was found that a graphene-like ordered nanostructure is adventitious for $\mathrm{ODH}$ of alkanes but a disordered polymer structure acts as access blocker to the active carbon deposit.

We further exclude graphene\{Rao, $2009 \# 2055\}$ from this discussion as too little of catalytic chemistry of genuine graphene is known. This refers to the catalytic action of systems\{Peres, 2006 \#3008;Radovic, 2005 \#2871\} containing strictly one carbon sheet. Such systems are, in addition, not available in forms amenable to catalysis. It was stated that this may change should for example electro-catalytic applications $\{\mathrm{Liu}, 2010$ \#602\} with supported graphene sheets become attractive. A chemically stable precursor system is graphene anchored in carbon nanotubes. As attractive as such systems may be electronically, the graphene units act as effective transport limitations for atoms and 
ions $\{\mathrm{Xu}, 2010 \# 1064\}$ and decrease the material conversion efficiency quite substantially. This was quantified for electrochemical Li ion storage but applies likewise for other potential chemo-catalytic applications. These statements would not preclude the application of "catalytic graphene "as chemically selective element in sensor applications where mass transport is no a limiting factor.

The application of graphitic carbon as catalyst support is well-known AAtamny, 2001 \#2710;Ma, 2001 \#2835;Toth, 2005 \#2831;Volta, 1979 \#2137;Xu, 2006 \#2829\}and has widely been studied. The field is mainly concertned with graphoitic carbon in the forms of black carbon and graphite nanofibers. Low surface area well-orderwd graphite is not a good support, as the interaction of metal species as well as of molecular reactants is weak with genuine basal planes\{Porte, 1988 \#2955\} of graphite. Results are poor fixation of metal species\{Atamny, 2001 \#2710;Baker, 1995 \#2840;Baker, 2004 \#306;Paal, 2005 \#2675\} and low sticking coefficients\{Marsh, 1974 \#3015\} of reactants. This is completely different on the infrequently abundant prismatic faces where dangling bonds and their covalently bonded terminating atoms form excellent sites of interaction with metal species. A classic example of this is the Ru.C catalyst used for ammonia synthesis\{Kotarba, 2003 \#3519;Kowalczyk, 1996 \#3521;Masthan, 1995 \#3538\} \{Guraya, 2004 \#3195\} where at great length a special graphitised form of carbon is produced as unique support\{Groszek, 1991 \#3272\} such as to expose multiple step structures on the basal planes acting as anchoring sites for the active Ru metal.

The high surface area carbons with less-well known surface electronic structure\{Pigamo, 2002 \#2921;Albers, 2001 \#3304;Smith, 1995 \#3312;Boehm, 1994 \#2903;Werner, 1993 \#2772;J.B. Donnet, 1993 \#3134\} are popular and effective supports metal species with wide uses in hydrogenation\{Besson, $2005 \# 2917$ \} and liquid phase\{Ehrburger, 1976 \#3021;Matatov-Meytal, 1998 \#3275;Ahumada, 2002 \#2888;Xu, 2006 \#2829;Herein, 1997 \#2742;Burmeister, 1993 \#2944;Gurrath, 2000 \#2929\} catalysis. Several reviews\{Radovic, 1997 \#2938;Rodriguez-Reinoso, 1998 \#2935\} cover this field. The ambiguous role of carbon as support, modifier and site blocker on technical hydrogenation catalysts was described\{Albers, 1994 \#2943;Albers, $2001 \# 2542\}$ in multi-method studies. The high stability of carbon against hydrolytic attack in aqueous media creates interest on carbon supports for biomass processing\{Shabaker, 2003 \#3288\}\{Chheda, 2007 \#2201\} occurring under hydrothermal synthesis conditions. 
Much work has been devoted to chemically activating the basal plane by oxidative functionalisation\{Hueso, 2007 \#3261;Razumovskii, 2007 \#3369;Giannozzi, 2003 \#76\}. The result is a pitted basal surface\{Blyholder, 1959 \#3034;Thomas, 1965 \#3037;Schlögl, 1990 \#839;Schlögl, 1992 \#812;Nowakowski, 1992 \#3243\} with acidic functional groups at the newly formed edges\{Henschke, 1994 \#92;Radovic, 2005 \#2871\}. Such structures may hold metal species in place as long as the supporting structures are not removed either thermally at moderate temperatures of about $473 \mathrm{~K}$ and above or chemically by spill-over of reactive species such as hydrogen for hydrogenation catalysts with Pt and $\mathrm{Pd}$ as active forms or oxygen with $\mathrm{Cu}$ and $\mathrm{Ag}\{\mathrm{Xu}, 2006 \# 2829\}$ as selective oxidation catalysts for epoxidation reactions at much lower temperatures\{Pigamo, 2002 \#2921\}\{Atamny, 1999 \#2815;Atamny, 2001 \#285\} than the thermal degradation of the functionalised artificial defects. (gallezot, atamny)

In this situation the synthesis of carbon-supported catalysts largely reverted to activated carbon materials with multiple rough and porous structures giving mechanical fixation to oxophilic metals such as Pd,Pt (paal?, reviews tierkohle)and with the chance to introduce many hetero-elemental defects\{Stohr, $1991 \# 216\}$ in the rough surface allowing to create local centres of electron density(stör boehm) holding electrophilic elements such as Ag, Au (haruta, likolobov) or oxides\{Trunschke, 2000 \#1661\}\{IllanGomez, 1999 \#2882\} as NP\{Okhlopkova, 2000 \#2927;Gurrath, 2000 \#2929\}\{Haruta, 1997 \#1114\} in place.

The situation changed drastically with the advent of curved sp2 carbons represented by the families of fullerenes \{Werner, 1992 \#2782;Werner, 1993 \#2772;Homann, 1998 \#2932;Guldi, 2000 \#84;Goldshleger, 2001 \#3047\}(refs including rs) and later of CNT, CNF systems. From now on a new bonding mechanism could be used. These families of NC have in common to exhibit an electronic structure different from that in flat graphene. The curvature topology introduces a lifting of the degeneracy of the “aromatic" fully delocalised\{Stephan, 1996 \#214;Hirai, 1995 \#3090\} valence electrons. At certain points in the geometric surface electron localisation\{Thomas, 2008 \#220;Carlsson, 2006 \#38\} (refs!!, plot of bonding and scheme of localization?) occurs giving rise to the conversion of aromatic "bezenoid" \{Guldi, 2000 \#84;Clar, 1981 \#385;Smith, 1995 \#3312\}(Clar) bonding properties to olefinic bonding properties. Covalent bonds between carbon as support and adsorbates or metal particles are now possible transforming the carbon into a large ligand. A kind of metal-organic 
coordination chemistry becomes possible with strong and tuneable carbon-metal interactions and hence much better support properties. This was realised first with $\mathrm{Ru}$ as active metal on fullerenes\{Braun, 1997 \#356;Nowitzke, 1997 \#665;Wohlers, 1996 \#996\} followed by other fullerene-metal systems used as catalysts\{Sherigara, 2003 \#3042;Goldshleger, 2001 \#3047\}.

Carbon naotubes and carbon nanofibers were found to be excellent supports. In these materials several advantages of carbon as support combine into a highly attractive property profile compensating for the high price of such supports. The advantage of bent sp2 /sp3 mixed hybridization with its potential for electron localization comes with a micropore-free support that cannot be hydrolyzed in corroding environment and is due to its surface perfection rather immune against activated reagents such as hydrogen and oxygen. These advantages can only be exploited if the surface of the nanocarbon is free of pyrolytic debris covering often nanocarbons obtained from CVD processes. Effective purification routines are available, whereas the often recommended "cleaning" in nitric acid is not a good strategy \{Rinaldi, 2010 \#733\}. In additon it is possible by "doping" the nanocarbon for example by nitrogen\{Stohr, 1991 \#216\}\{Arrigo, 2010 \#1059\}, to modify its electronic structure in a still quite unknown way\{Arrigo, 2008 \#16\}\{Toebes, 2003 \#3343\}\{Maldonado, 2006 \#3240;Belz, 1998 \#2737\} that makes the carbon even more stable against chemical attack. A typical example is the use of Pd/CNF in the synthesis of $\mathrm{H} 202$ from the elements\{Abate, 2010 \#2576\} that both in their activated forms extremely corrosive to conventional carbon.

\section{Case studies}

The following examples illustrate the use of NC in heterogeneous catalysis. As discussed above we will first review some applications of $\mathrm{NC}$ as metal-free catalysts and then revert to studies where NC has is used as special support for metallic nanoparticles. The case studies should illustrate the application potential but also the extent of synthetic and analytical effort necessary to undoubtedly trace back the observed catalytic function to the properties of the NC. It is intended to illustrate that the application examples do not follow an empirical approach of testing a novel material for some application but rather an concept-driven approach based on the understanding of the structural features and resulting chemical consequences. 
The as-received NC need some pre-treatment or functionalization to bring about their true potential for a given application. In some cases this occurs autogenously during reaction, in many other cases we need modifying the surface by chemical procedures prior to applying a metal catalyst or exposing the surface to reactive gas atmospheres. An accurate determination of surface properties before and after functionalization is a pre-requisite for useful studies. This is not explicitly repeated in every example discussed below.

\section{NC in styrene synthesis}

The synthesis of styrene from ethyl benzene is a large-scale industrial process with over 20 million metric tons production per year. The current production technology\{Cavani, 1995 \#242\} uses dehydrogenation of ethyl benzene over iron-oxide based catalysts. Besides the drawback of a thermodynamic limitation of the conversion (between 50-60 $\%$ depending on operation conditions) and the high energy input required $(\Delta \mathrm{G}=124.9$ $\mathrm{kJ} / \mathrm{mol}$ ) a severe process drawback is the need to co-feed about a tenfold excess of steam at operation temperatures of $873 \mathrm{~K}$ or more. Could one convert the dehydrogenation to an oxidative dehydrogenation with consecutive formation of water following the dehydrogenation step then the energetic balance would grossly change to $(\Delta \mathrm{G}=-116 \mathrm{~kJ} / \mathrm{mol})$ and no steam would have to be co-fed. A variation of this is the autothermal dehydrogenation where it is assumed that the oxygen fed to system burns the hydrogen of a conventional dehydrogenation process and creates in the bed the necessary thermal energy for the dehydrogenation. Such a process would benefit from a microstructured reactor with its optimum internal heat transfer properties. NC materials although not yet used could play an important role as they can

An in-depth study of the nature\{Muhler, 1992 \#209; Weiss, 2002 \#241;Weiss, 1998 \#168\} and mode of operation\{Huang, 2005 \#97;Joseph, 2000 \#149;Ketteler, 2002 \#133;Kuhrs, 2001 \#140;Shekhah, 2004 \#118\} of the technical promoted iron-oxide catalyst showed that the active phase is either a potassium ferrate overlayer or a carbon overlayer covering in both cases densely the iron oxide. Evidence was found and confirmed by reactor modelling\{Schule, 2007 \#71;Schule, 2005 \#104\} that a highly active state of the catalyst is related to iron $3+$ ions in a matrix and a low-active state is due to a specially ordered\{Shekhah, 2004 \#118\} form of carbon. Both active sites are deactivated by coverage with disordered carbon coming about from reaction with reduced iron oxide\{Huang, 2005 \#97;Shekhah, 2004 \#118;Weiss, 1998 \#168\} sites. 
These facts promoted the use of carbon as catalyst\{Drago, 1994 \#250;Pereira, 2001 \#244;Pereira, 2000 \#245;Pereira, 1999 \#247\} for ODH of ethyl benzene. Several attempts failed due to rapid deactivation of the catalyst caused by deposition of disordered\{Drago, 1994 \#250;Fiedorow, 1981 \#254;Pereira, 2004 \#243;Pereira, 2001 \#244;Vrieland, 1991 \#253\} carbon. The micro porosity of the used carbon led to polymerisation of the styrene causing unwanted carbon deposition. Nanocarbons can be made free of micro pores and should thus not deactivate\{Pereira, 2004 \#243\} so fast. In addition, specific active sites may be implemented on NC by functionalization and by controlling the surface ordering of the NC as was discussed in previous sections of this work.
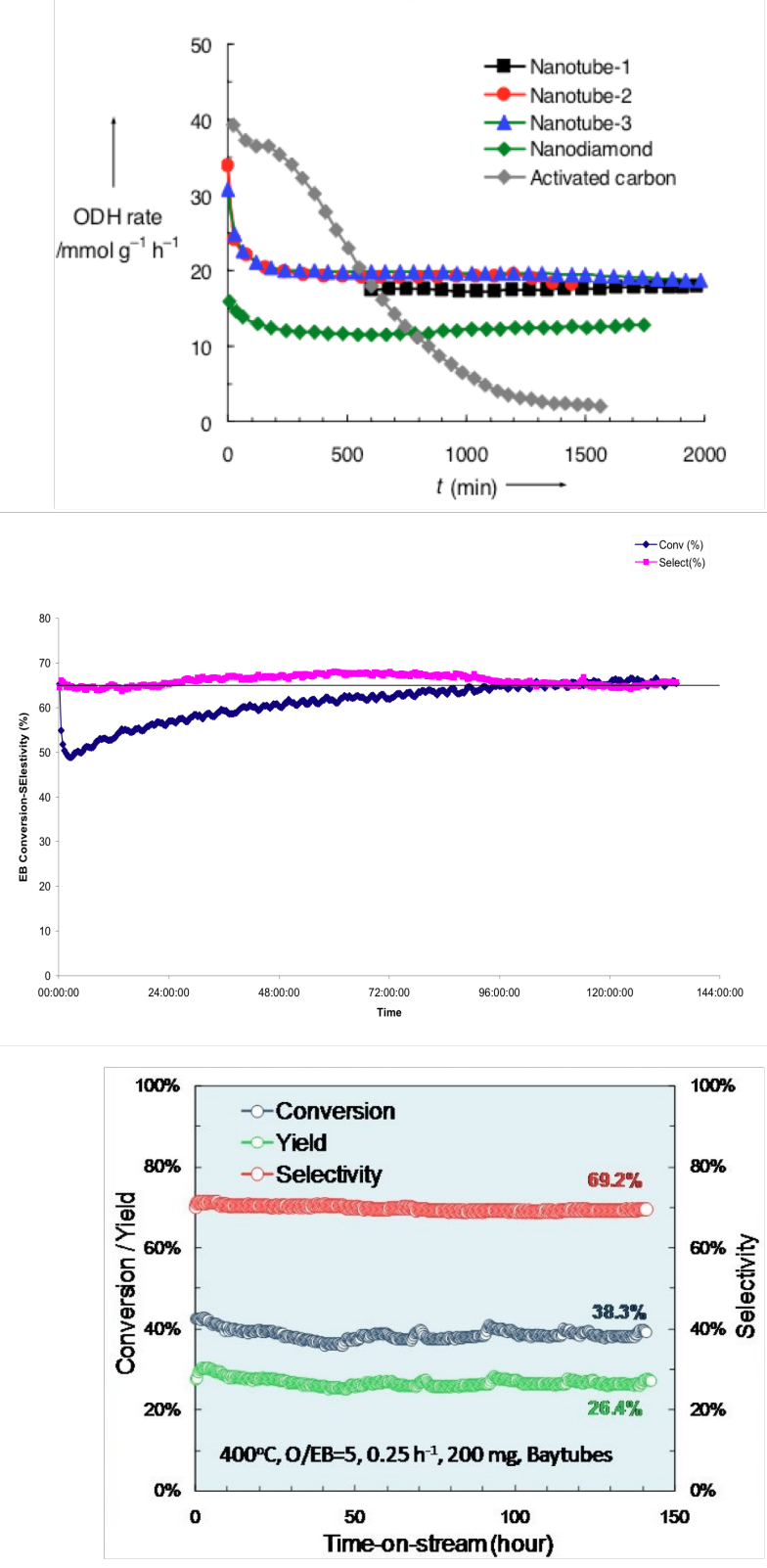
Figure 8: Summary of the performance of $N C$ in the ODH of ethyl benzene. (A) summarizes\{Zhang, 2007 \#28\} the activity and stability of NC in comparison of activated carbon, (B) presents the initial section of a long-term stability test\{Delgado, 2010 \#154\} and $(C)$ shows that under similar conditions than (B) also commercial abundant CNT are active and stable catalysts.

First it was shown\{Keller, 2002 \#132;Mestl, 2001 \#141\} that MWNT and CNF are active and selective catalysts in ethyl benzene ODH. Figure 8A shows that activated carbon\{Dangsheng, 2007 \#78\} is a good catalyst for ODH of ethyl benzene but it undergoes rapid deactivation to complete loss of activity within 24 time on stream. NC both in the form of nanotubes and nanodiamond that converted to highly curved sp2 nanocarbon shells\{Roddatis, $2002 \# 138\}$ are, however, active and stable catalysts. In a scale-up experiment documented in Figure 8B about 100g of well-ordered CNT were used\{Delgado, $2006 \# 84 ; \mathrm{Su}, 2009 \# 26\}$ as catalyst in a test shown here for $150 \mathrm{~h}$ that lasted without detectable deactivation for 32 days. In Figure $8 \mathrm{C}$ we document that is also possible\{Zhang, $2009 \# 42\}$ to use large-scale commercial BAYTUBE materials\{Tessonnier, 2009 \#22\} for the ODH of ethyl benzene without applying any pre-treatment for removing detrimental catalyst particles or optimizing the CNT surface structure.

Early conjectures\{Mestl, 2001 \#141\} followed by in-situ experimentation\{Macia-Agullo, 2005 \#98 \} assumed that a strongly basic form of oxygen functional groups should be the active sites. It was assumed that di-carbonyl groups in resonance with the pi system of the NC and with themselves (quinoidic) could be candidates for the active sites. Final proof for this conjecture came from the synthesis of a molecular phenanthreneqinone cyclotrimer analogue of such groups and the confirmation\{Zhang, 2009 \#772\} that this species is an excellent catalyst both with respect to activity and stability operating at substantially lower temperature (595 K) than the NC ( $673 \mathrm{~K}$ ) catalysts with about two orders of magnitude higher rate. 
A series of CNT from the CVD method with relatively high ordering\{Delgado, 2010 \#154\} was subjected to a heat treatment for an increase of surface ordering. The better surface ordering is an effective control for the abundance of oxygen functional groups being located at defect sites. An order parameter was derived from the integration of the quantitatively measured (002) reflection of the ordered stacks of the CNT. Table 1 shows the good correlation of the order parameter to the amount of oxygen found after $100 \mathrm{~h}$ of reaction by quantitative elemental analysis, which agreed well with a simultaneous determination by XPS. TEM verified that no significant deposition of carbon occurred during catalysis which may interfere with the relation of CNT ordering to the oxygen content.

Table 1: Selected parameters of CNT for ODH of ethyl benzene

\begin{tabular}{|l|l|l|}
\hline Treatment Temperature (K) & Order parameter & Oxygen content $\left(\mathbf{m m o l} / \mathbf{m}^{2}\right)$ \\
\hline 1000 & 0.36 & 5.3 \\
\hline 1250 & 0.47 & 4.7 \\
\hline 1400 & 0.57 & 2.5 \\
\hline 1800 & 1.00 & 0.45 \\
\hline
\end{tabular}

In Figure 9 the rate of ethyl benzene conversion and the selectivity to styrene are plotted against the oxygen content for testing parameters of $673 \mathrm{~K}$, oxygen/EB ratio 5:1, WHSV $1.0 \mathrm{~h}^{-1}$ representing rather harsh conditions at conversions of $50 \%$ and lower to minimize transport limitations. The Figure reveals a good correlation between oxygen content and kinetic parameters substantiating the identification of active sites with oxygen functional groups. 


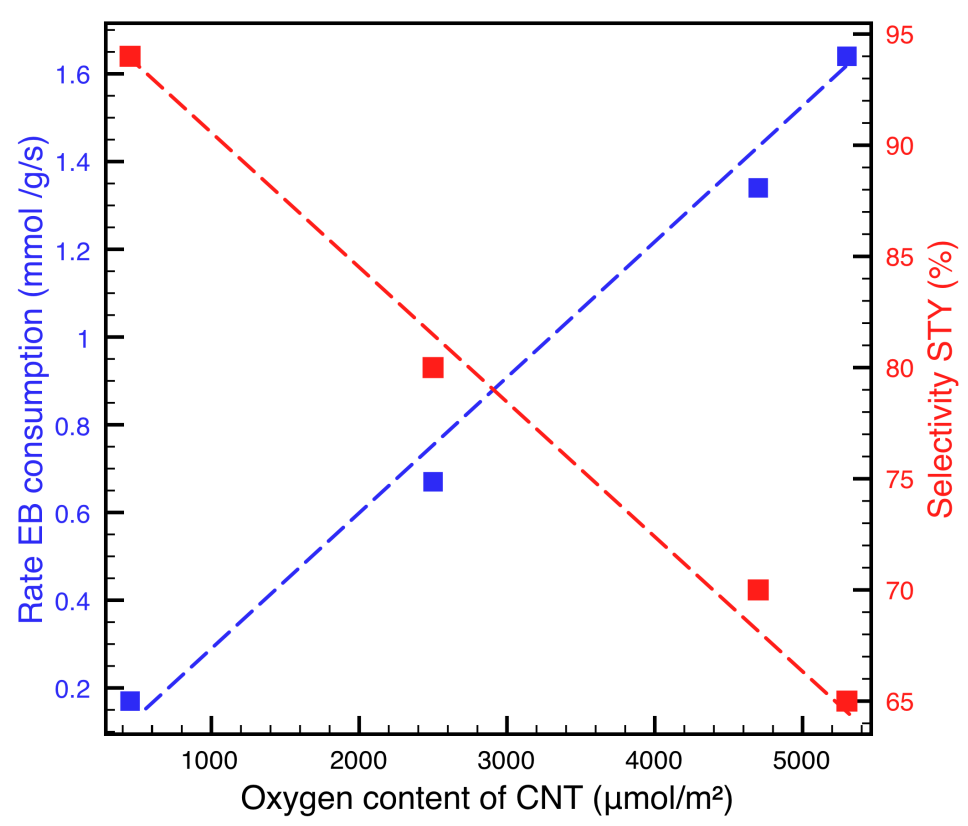

Figure 9: Relation of kinetic parameters of ODH of ethyl benzene with the oxygen content of CNT catalysts of various surface ordering. Data taken from ref.\{Delgado, 2010 \#154\}.

One may further conclude that for the reaction conditions chosen an optimum oxygen site density on the order of $3 \mathrm{mmol} / \mathrm{m}^{2}$ would be adequate for balancing the reaction rate with useful selectivity. A productivity of about $1 \mathrm{mmol}$ STY/g CNT and second is a quite acceptable value for a partial oxidation reaction and at an attainable selectivity of $90 \%$ a recirculation operation of the dry feed may be an adequate engineering realization.

The fact that there is an optimum charge of the catalyst with oxygen functional groups is proof that more than one oxygen group interferes with the overall reaction and that at least one type of species is counterproductive for selective oxidation. As expected, the rate of ethyl benzene conversion increases with increasing oxygen abundance per unit surface area. The reaction does, however, not stop after styrene formation but can lead to the formation of undesired non-selective oxidation products such as benzene, toluene and $\mathrm{CO}_{2}$. This second consecutive reaction sequence also occurs on oxygen sites as it is also increasing in probability with increasing total oxygen abundance. The observation of an optimum is evidence for the presence of different oxygen groups acting as active sites but counted together with the elemental analysis of the oxygen content. This is not surprising keeping in mind the diversity of surface functional groups discussed above. 
It is instructive to compare the performance of the CNT system to oxide catalysts more frequently used for selective oxidation reactions. Unfortunately, as the reaction conditions are different and incompletely described in the literature, only a coarse comparison is possible using kinetically less useful parameters. Table 2 reports some data

Table 2: Selected performance data of different catalyst families for ODH of ethyl benzene

\begin{tabular}{|l|l|l|l|}
\hline Catalyst & Conversion (\%) & Selectivity (\%) & Ref. \\
\hline VxOy magnesia & 25 & 80 & $\{$ Chang, 1995 \#249\} \\
\hline Vanadia /AC & $50^{*}$ & 80 & $\{$ Sakurai, 2000 \#246\} \\
\hline La/AC & $42^{*}$ & 90 & $\{$ Sakurai, 2000 \#246\} \\
\hline CNT immobilized & 35 & 80 & $\{$ Delgado, 2007 \#77\} \\
\hline CNT optimized & 65 & 95 & $\{$ Zhang, 2007 \#75\} \\
\hline
\end{tabular}

* strong deactivation within less than $24 \mathrm{~h}$

The values for the CNT being near the values also shown in Figure 8 compare favourably with those of typical metal oxide systems such as vanadia. The comparison gets even more favourable for the carbon system when we consider the stability of the performance. It is remarkable that a material having no transition metals at all in it, "a metal-free catalyst" operates in a reaction requiring redox activity at least as well as the typical metal-based redox-active systems. It is further seen that the often-claimed necessity to involve lattice oxygen\{Arena, 1997 \#985;Chen, 2002 \#986;Grasselli, 2002 \#987;Ovsitser, 2009 \#988;Ozkan, 2005 \#989\} for selective reaction is not a critical property for selective oxidation reactions. Lattice oxygen is thought to be bonded strongly the catalyst surface and should thus oxidize less vigorously\{Grasselli, 2002 \#987\} than activated gas phase oxygen. Alternatively\{Abd Hamid, 2003 \#991\} selective oxidation reactions may be considered as processes requiring two types of activated oxygen namely nucleophilic (strongly bound) oxygen for $\mathrm{C}-\mathrm{H}$ activation and electrophilic (weakly bound) oxygen for oxygen insertion reactions. Active sites for oxidation reactions of hydrocarbons require in this picture two different bonding sites for oxygen symbolized chemically with double bonded and single bonded oxygen.

In such a picture it becomes clear why CNT may be good catalysts for ODH of ethyl benzene. The reaction should best proceed if the active site contains nucleophilic oxygen 
only as then only the dehydrogenation step would occur. After desorption of the styrene molecule activated oxygen should react with the protonated nucleophilic sites and form water plus the required energy.

Nanocarbons can provide with di-ketonic functions the sites of nucleophilic oxygen for dehydrogenation. Thus it no surprise, that oxygenated CNT are active catalysts. More difficult is the understanding of the second part of the reaction involving the reoxidation of the "alcoholic" functions. The general part of this text delivers the explanations of how this reaction can occur and why CNT are much better suited than planar or defective carbon to perform oxygen activation in a catalytic way. From this we would conclude that ODH is not a single reaction but rather a consecutive reaction of a dehydrogenation step at ketonic groups and water-forming oxidation step using reactive atomic oxygen not directly attached to the active site but diffusing on the basal planes of sp2 carbon. Such a mechanism has been suggested based on phenomenological observations already by Emig\{Emig, 1983 \#1003\} and is now the generally accepted\{Serp, 2009 \#994\} mechanism. For an illustration of the process see\{Serp, 2009 \#994;Maksimova, 2000 \#402\} the literature.

It was possible to put experimental evidence on this both by observing the dynamic change of the oxygen functional groups as function of catalytic activity by photoemission experiments\{Macia-Agullo, 2005 \#26\} and by synthesizing a model compound.

The concept of the model compound and its catalytic function are shown in Figure 10 
(7)

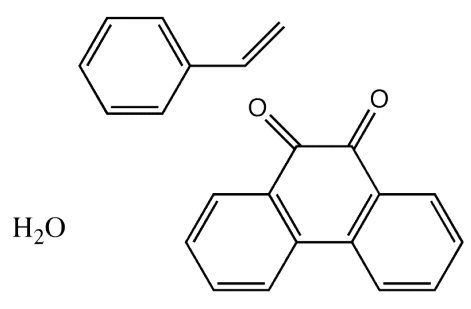

(6)

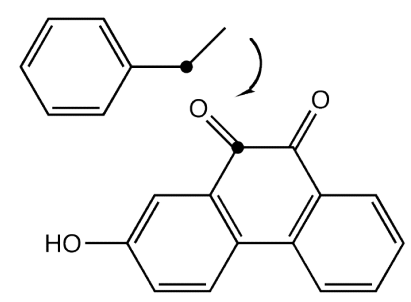

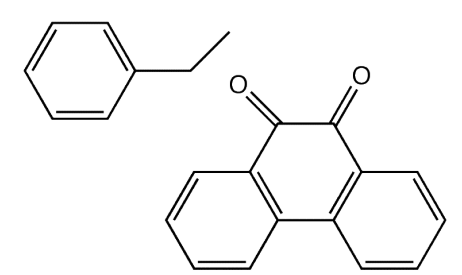

(1)

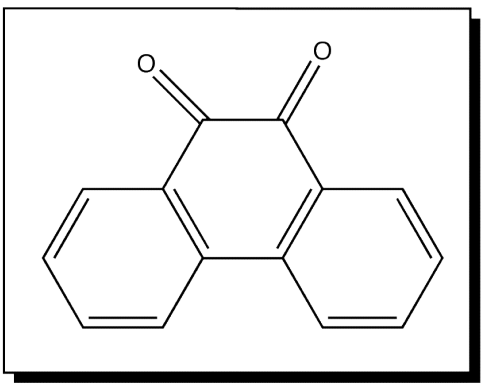

(5)

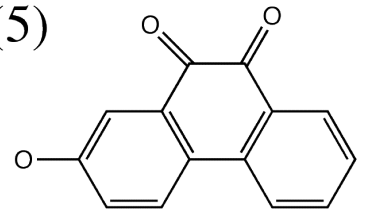

$\mathrm{H}_{2} \mathrm{O}$

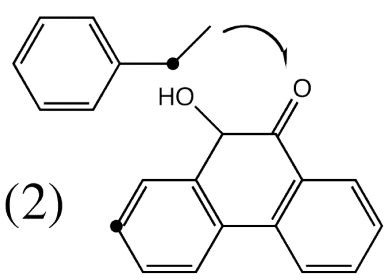

(3)

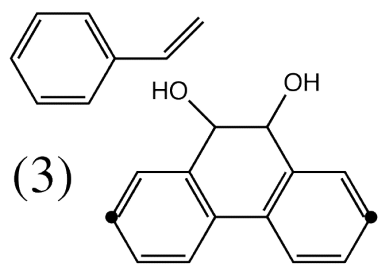

(4)

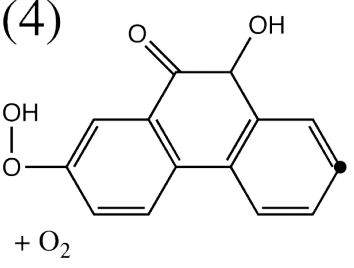

Figure 10: A concept of an active site for oxidative dehydrogenation. For the individual numbers indicating reaction steps see text. The central feature symbolizes the concept of the active site.

For the interaction of EB with the active site (1) we assume that the catalyst is loaded with pre-adsorbed EB in a suitable configuration that is not blocking the active site. The reaction order of 0.5 found\{Zhang, 2007 \#71\} for EB on several carbon catalysts is in line with a sufficient presence of adsorbed educt during reaction conditions. In step (2) the most difficult step is performed namely the activation of the alkyl group through formation of a di-radical structure. Such a reaction requires the action of a nucleophilic oxygen (the $\mathrm{C}=0$ ) and of an electron acceptor (the aromatic carbon structure). In step (3) the relatively facile reduction of the quinoidic structure and the formation of the styrene molecule occur. The kinetic analysis of Zhang\{Zhang, 2007 \#771\} showed no evidence for site blocking of the reaction product.

Reaction steps (4) and (5) illustrate the re-oxidation steps of the active site. The occurrence of a peroxo- or radical oxygen structure owing to the fact that 4 electrons or two ethyl benzene molecules are required to activate one di-oxygen molecule gives the explanation for the finite selectivity of the reaction; it is unavoidable that electrophilic partly activated oxygen co-exists at finite conversions (see reaction step 6,7 in Figure 
10) at the active sites. Minimization of the interference of electrophilic oxygen (step 5 in Figure 10) with the desired operation of the nucleophilic sites (steps 2, 6 in Figure 10) is achieved when the abundance of electrophilic oxygen is minimized by a sufficient localization of electrons from the ethyl benzene oxidation stored on the catalyst. The molecular nature of the model catalyst\{Zhang, 2009 \#772;Zhang, 2009 \#772\} and strongly curved NC structures are thus preferred realizations of the system limited only be the increasing stability of electrophilic oxygen on curved carbon as evidenced\{Wohlers, 1996 \#1007;Werner, 1992 \#1006\} by the auto-oxidation of C60 fullerene. This explains also the good performance\{Dangsheng, 2007 \#20;Kuznetsov, 1994 \#361;Roddatis, 2002 \#21\} of the highly curved and poorly long-range conducting graphene overlayers on naodiamond.

\section{$\mathrm{NC}$ in alkane ODH}

The reaction sequence suggested in Figure 10 is similar to mechanisms discussed over oxide catalysts\{Feyel, 2006 \#199\} for non-activated alkanes\{Chen, 2002 \#986;Chen, 2000 \#1009\} such as propane. The critical step is there also the formation of a di-radical first intermediate\{Rozanska, $2007 \# 1008\}$ as C-H activating step. It should thus be possible to activate these non-reactive molecules selectively over NC systems. It is expected that with decreasing stability of the radical first intermediate the reaction should become increasingly more difficult from butane over propane to ethane. For the same reason of decreasing stability of activated intermediates the propensity to deep oxidation should decrease in the same order offering the chance of a finite productivity in ODH. It was possible to achieve ODH of butane\{Zhang, $2008 \# 768\}$ over clean NC catalysts and over borate or phosphate modified CNT also for propane\{Frank, 2009 \#216;Rinaldi, 2010 \#538\} and for ethane\{Frank, \#998\}.

The technical dehydrogenation of butane over vanadium oxide-based catalysts occurring under similar harsh conditions than that of ethyl benzene was found\{McGregor, 2010 \#32\} also to be operating on a shell of carbonaceous overlayer rather than over a bare oxide catalyst, which is active and selective but not stable at operation conditions. It was further shown that CNF-like structures identified with the novel technique of $\mathrm{THz}$ spectroscopy are the active species. These species can further catalyze the pure dehydrogenation at elevated temperatures. It is suggested that under 
the DH conditions the dissociative dehydrogenation of the phenolic intermediates (step (3) in Figure 10) will require the high temperature, as the ODH reaction of NC can easily occur at $250 \mathrm{~K}$ lower temperature.

The reaction of butane ODH over NC was further used to confirm the character of the NC as metal-free catalyst. There is worry that the transition metal traces present in nanocarbons from their synthesis may be catalytically active. It can indeed always be verified by TEM\{Zhang, 2008 \#768\} that some metal particles remain in the NC even after deliberate washing as these particles are encapsulated by graphitic carbon and remain thus protected from e.g. acid attack. Under ODH conditions they may get liberated and then act as catalyst. It was shown that this is not the mode of operation of the NC ODH catalysts. A series of NC catalysts was prepared\{Zhang, 2008 \#768\} with increasing amounts of supported iron oxide to mimic the state of an iron catalyst after oxidative liberation. The unexpected consequence was that the catalysts lost substantial performance with increasing loading as the metal oxide was only catalyzing combustion and no selective reaction. It is noted that no significant increase in conversion of the alkane occurred with the metal-loaded systems indicating that at the low temperature of operation $(650 \mathrm{~K})$ the metal oxide was intrinsically less active for alkane activation than carbon. It is noted that this particular behaviour is not a property of any carbon but only of curved and oxygen-functionalized NC systems.

The ODH reaction over CNF was also used to verify the role of ketonic oxygen functional groups as active sites and to verify the postulated existence of other oxygen functional groups not related to the dehydrogenation reaction path. In Figure 11 a set of in-situ XPS experiments is shown. The working catalyst was investigated for its abundance of oxygen functional groups under dehydrogenation (Figure 11 top C) and oxidative dehydrogenation (Figure 11 top A) conditions. The in-situ XPS showed two groups of oxygen functions being present under reaction conditions. No attemptw as made to unravöe the exact distribution of species due to the ambiguities discussed above. The low binding energy component contains the expected ketonic groups, whereas the high bindinhg energy peak summarizes alkoholoc and electrophilc oxygen species. Before the reaction sets in both familines of species are equally abundant. From the dehydrogenation experiment it occurs that the reaction consumes the ketonic functiona and is eventually deactivating as it looses its active sites. Regeneration is possible but was not deeper investigated. Under ODH conditions we record in the presence of oxygen 
(visible in the XPS spectra by its gas-phase signal) a prevalence of the ketonic groups. This is a property of the CNT system, in most microporous and defective carbons the C-O signal is dominating at these temperatures.

In order to reduce the sensitivity of the CNT to auto-oxidation through electrophilic oxygen we protected the NC by impregnation with small amounts of phosphate decorating the acidic groups at defect sites and thus inhibiting the facile further attack by electrophilic mobile oxygen. This protection principle\{Lizzio, 1990 \#388\} is known since long in carbon material science and also works here. It can be seen from Figure 11(top B) that the presence of the phosphate has no discernible effect on the distribution of active oxygen, its own oxygen signal is covered under the electrophilic signal group.

The stable operation of the propane ODH over CNT can be used to substantiate the equivalent operation of metal-free catalysts and metal-oxide systems. This is seen as indication that the reaction proceeds via the same critical intermediate. Not only the wanted main reaction but also the side reactions of electrophilic attack seem to be rather similar in both cases as can be seen from the stable performance of both systems with almost identical catalytic performance. The satisfying selectivity is due to the moderate temperature of $650 \mathrm{~K}$ used in the experiment where despite an excess of oxygen over the propane the side reactions are almost tolerable. It is noted that the overall performance is good for this difficult reaction\{Chen, 2002 \#986;Dinse, 2008 \#1010;Dinse, 2009 \#1011;Ovsitser, 2009 \#988\} with many sophisticated oxide catalysts not performing to the level indicated in Figure 11.

The conjecture about a similar reaction over oxide and carbon catalysts is substantiated\{Frank, 2009 \#216\} by the observation that on all catalysts (oxide, bare carbon, phosphate and borate modified) the apparent activation energy was the same. Its value of $130( \pm 5) \mathrm{kJ} / \mathrm{mole}$ was about the same as for the activation of oxygen when the NC was unmodified. For the vanadia reference catalyst and for the modified NC the oxygen activation energy was about $40 \mathrm{~kJ} /$ mole higher indicating that a kinetic barrier for the oxygen activation regulates the availability of activated oxygen and thus contributes to the minimization of the abundance of electrophilic oxygen. This has a price to be paid in rate of conversion. With increasing abundance of modifiers between $0.01 \mathrm{wt} \%$ and $0.5 \mathrm{wt} \%$ the rate falls by a factor of 5 from $5 \mathrm{mmol} / \mathrm{g} / \mathrm{h}$. The fact that the 
propane activation energies do not change with presence or loading of the modifiers is a good indication that the reaction does still take place at the same oxygen functionalized sites shown in Figure 10. With respect to the mode of operation of the vanadia catalyst it can be concluded that this system should also have two $\mathrm{M}=0$ bonds and can thus not be a monomer\{Rozanska, 2007 \#1008\} but should be oligomeric\{Cavalleri, 2009 \#1012\}.

The pronounced effect of the modifiers on the performance\{Frank, $2009 \# 216\}$ of the system and the identity of the alkane activation reaction over modified, unmodified and oxidic systems suggest that the oxygen activation reaction is controlled by the modification. It is widely assumed\{Marsh, 1974 \#407;Marsh, 1965 \#411;Atamny, 1992 \#25;Atamny, 1992 \#26;Centi, 2009 \#115;Grushko, 1996 \#248;Henschke, 1994 \#272\} that oxygen activation on carbon required defects in basal planes or the edge sites of a graphene layer. This also true for not too highly bent graphenes (see above) we observe thus\{Frank, \#999;Rinaldi, 2010 \#538\} a correlation between defect density and oxygen activation activity. Passivating the defect sites being the active sites for oxygen activatiopn reduces thus the abundance of electrophilic activated oxygen. This species being chemically hydroperoxides, atomic oxygen or oxygen radicals are detrimental for the selectivity if in excess over the abundance needed to re-activate the phenolic hydrogenated sites of dehydrogenation (see Figure 10). This dual function of the electrophilic species can be seen in the changing formal reaction order of oxygen in ODH increasing\{Frank, \#998\} for ethane ODH from 0.1 for unselective non-modified samples to 0.5 for selective modified materials. The chemical nature of electrophilic oxygen as related to species that contain oxygen-oxygen bonds was substantiated\{Frank, \#999\} by SSITKA (steady state isotopic transient kinetics) experiments with oxygen isotope exchange. It was seen that modification of CNT with B and P both completely suppressed scrambling between the oxygen isotopes whereas unselective unmodified CNT did show rapid oxygen isotope scrambling.

With increasing process temperature these species begin also to burn the NC catalyst and thus create more defects which auto-catalytically leads first to loss of selectivity and at longer time on stream also to the loss of the catalyst. As there is carbon species in the feed the destruction of the catalyst may be self-repaired as the defects may well react with hydrocarbons to stabilize their dangling bonds. The need for modifying the NC against over-activation of oxygen at defects increases thus with the operation temperature of the process changing from $650 \mathrm{~K}$ for butane to $723 \mathrm{~K}$ for ethane. A 
quantitative model based upon kinetic studies\{Frank, \#999\} of oxidation of CNT with diluted oxygen reproduces well the observation that the critical temperature range is between $600 \mathrm{~K}$ and $700 \mathrm{~K}$ where the dilution of oxygen (chemical potential) decides over stability or moderate gasification being inacceptable for a catalytic process.

Attempts to capture the chemical nature or even the diversity of the defects by quantification of their TPD signatures were not successful. The quantitative determination of $\mathrm{CO}$ and $\mathrm{CO}_{2}$ being proxies for multiple (acidic) and single (basic) oxygen functional groups revealed drastic changes as reported in table 3.

\begin{tabular}{|l|l|l|l|}
\hline Groups & $0.5 \mathrm{mmol} / \mathrm{g} \mathrm{B}$ & $0.7 \mathrm{mmol} / \mathrm{g} \mathrm{P}$ & oxidized \\
\hline $\mathrm{CO}_{2}$ freh & 882 & 874 & 1474 \\
\hline $\mathrm{CO}_{2}$ used & 812 & 761 & 939 \\
\hline CO fresh & 34 & 1688 & 341 \\
\hline $\mathrm{CO}$ used & 403 & 2251 & 1500 \\
\hline $\mathrm{S} \mathrm{C}_{2} \mathrm{H}_{4}(\%)$ & 58 & 62 & 40 \\
\hline
\end{tabular}

Table 3: Selected values of oxygen functional groups from quantitative TPD analysis for NC catalysts in ethane ODH. Data taken from ref\{Frank, \#998\}.

The samples differ significantly both in content and in stability of oxygen functional groups. The catalytic performance as judged in ethylene selectivity at $5 \%$ ethane conversion is not sensitively correlated to any of the quantitative parameters. The conclusion is that only a small fraction of oxygen functional sites are active and that these sites are hidden in their profile under the desorption responses from many more not reactive species. The data in table 3 show clearly that the modifiers strongly influence the distribution of oxygen groups and that also their chemical effect is different for boron and phosphorous. From water and ammonia TPD experiments we can conclude that boronic acid stays more an acid whereas phosphate is barely present as acid forming ester groups with the oxygen functional groups. A systematic 
combination of chemical modification with thermal treatments is needed to better resolve the surface chemistry of the NC. It can be stated that in general well-developed NC with homogeneous shapes and minimal structural defects are good candidates for catalysis whereas highly disordered or strongly graphitized (graphene-like) varieties are less-well suited as they are too instable or too inactive. Single wall CNT are unsuitable as they react easily with molecular oxygen due to their strong curvature of the walls.
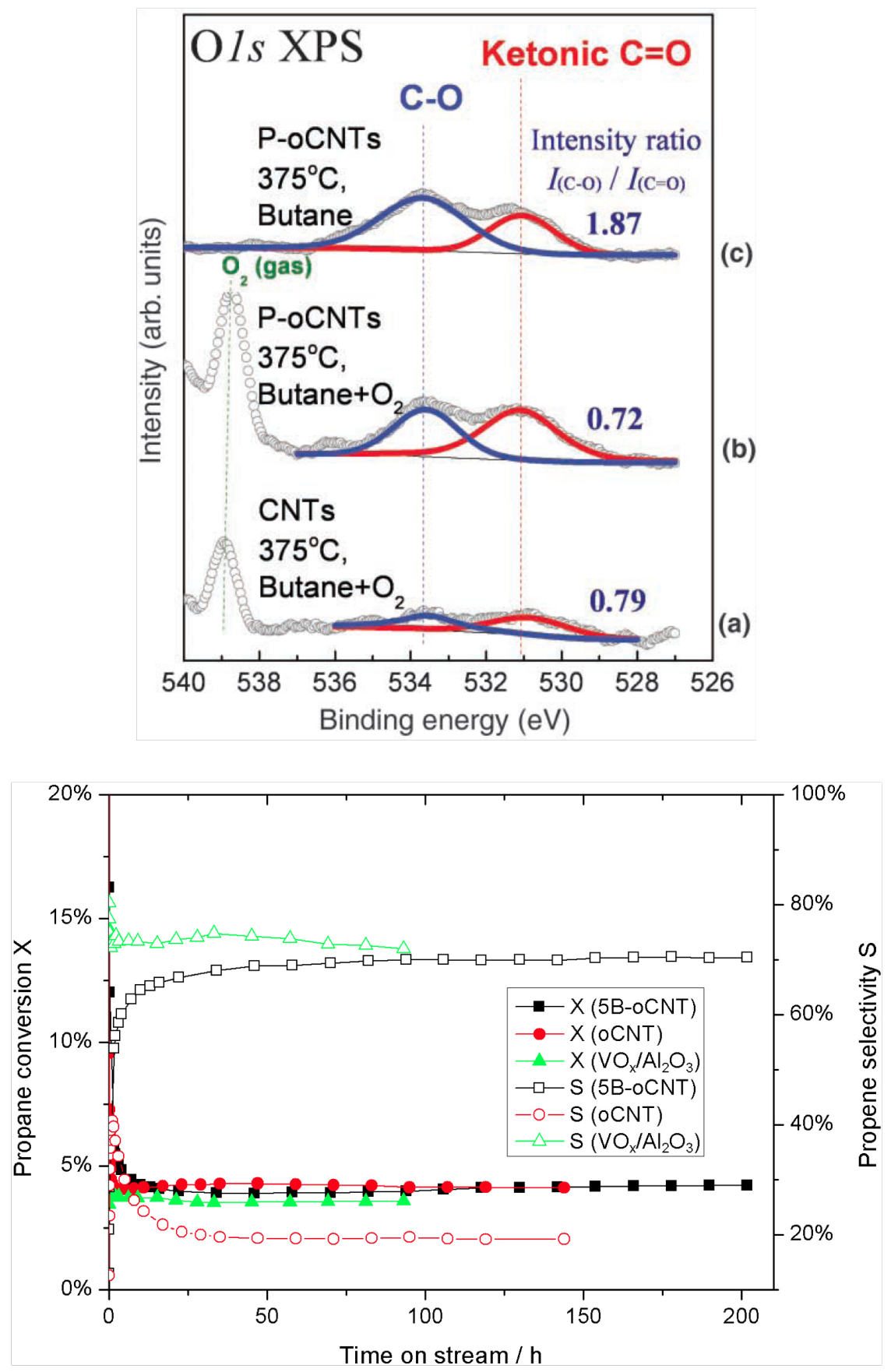
Figure 11: Top: in-situ XPS data of butane ODH\{Zhang, 2008 \#768\} under conditions indicated. Bottom: Comparison of performance of bare and boronic acid-modified CNT with a vanadia-alumina reference catalyst\{Frank, 2009 \#216\} in propane ODH.

\section{Supported NC systems}

This area of catalysis application is much more widely researched and many reports can be found in the literature that is quite comprehensively reviewed in the references given in the Introduction.

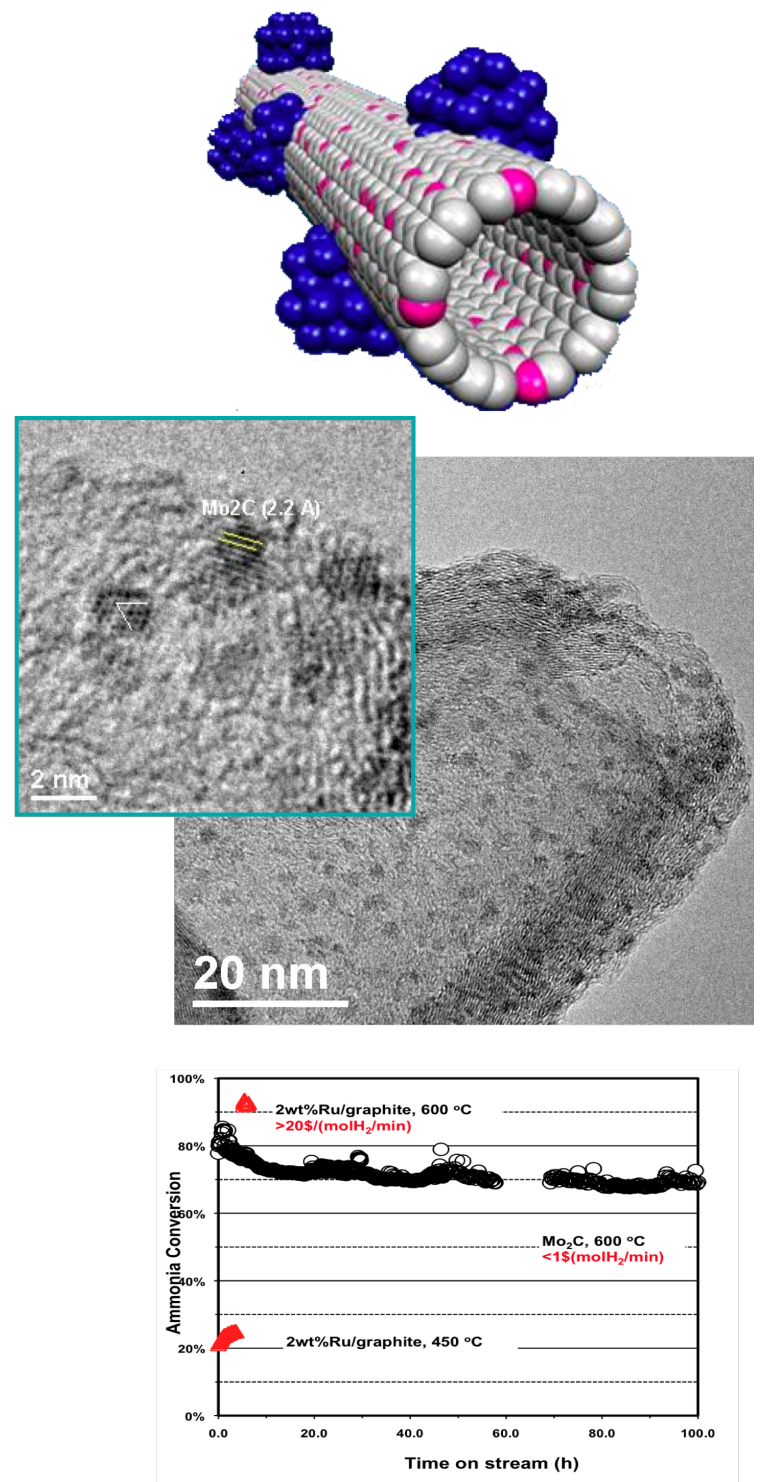

Figure 12 
